\title{
Metodología para la implementación de la gestión de riesgo en un sistema de gestión de calidad*
}

\author{
Methodology for the Implementation of Risk Management \\ in a Quality Management System
}

\section{Metodologia para implementar a gestão de risco em um sistema de gestão da qualidade}

Recibido: 15 de julio de 2019

Alfonso Doria Parra*

Universidad del Atlántico, Barranquilla, Colombia

Leandro Lópes Benavides**

Universidad del Atlántico, Barranquilla, Colombia

Marjorie Bonilla Ferrer ${ }^{* * * *}$

Universidad del Atlántico, Barranquilla, Colombia

Griselda Parra Cera ${ }^{* * * *}$

Universidad del Atlántico, Barranquilla, Colombia

Cómo citar este artículo: Doria-Parra, A., López-Benavidez, L., Bonilla-Ferrer, M. \& Parra-Cera, G. (2019). Metodología para la implementación de la gestión de riesgo en un sistema de gestión de calidad. Signos. Investigación en Sistemas de Gestión, 12(1), 123-135. DOI: https://doi.org/10.15332/24631140.5424

Artículo de resultado de investigación.

** Magíster en Calidad y Gestión Integral, especialista en Administración de Empresas, química farmacéutica. Bogotá, Colombia. Correo electrónico: adry.cardenas@gmail.com. Orcid: https://orcid.org/0000-0002-5980-0964

*** Magíster en Calidad y Gestión Integral, especialista en Administración de Empresas, química farmacéutica. Bogotá, Colombia. Correo electrónico: adry.cardenas@gmail.com. Orcid: https://orcid.org/0000-0002-5980-0964

*** Magíster en Calidad y Gestión Integral, especialista en Administración de Empresas, química farmacéutica. Bogotá, Colombia. Correo electrónico: adry.cardenas@gmail.com. Orcid: https://orcid.org/0000-0002-5980-0964

**** Magíster en Calidad y Gestión Integral, especialista en Administración de Empresas, química farmacéutica. Bogotá, Colombia. Correo electrónico: adry.cardenas@gmail.com. Orcid: https://orcid.org/0000-0002-5980-0964 


\section{RESUMEN}

El enfoque basado en riesgo es uno de los principales cambios de la nueva versión de la norma ISO 9001:2015, y exige de las organizaciones, además del compromiso de la alta dirección, establecer durante la planificación del sistema de gestión de la calidad acciones que le permitan determinar los riesgos y oportunidades con el fin de asegurar que el sistema de gestión de la calidad pueda lograr sus resultados previstos, aumentar los efectos deseables, prevenir o reducir efectos no deseados y lograr la mejora continua de sus procesos. En el presente artículo, se plantea una metodología genérica para administrar los riesgos estratégicos y operacionales de una compañía a partir de la comprensión del contexto y la identificación de las necesidades y expectativas de las partes interesadas. Por medio de una secuencia de etapas se plantea abordar los riesgos y oportunidades de una organización, partiendo de la identificación de las fuentes, causas y consecuencias que puede traer la materialización de un riesgo inherente, seguido de la valoración a través de un análisis cualitativo, cuantitativo, semicuantitativo o combinación de estos, de acuerdo con la herramienta que se desee utilizar. Posteriormente, teniendo el riesgo valorado se evalúa haciendo uso de las estrategias definidas para identificar los riesgos críticos y priorización de estos. Por último, continúa el proceso de tratamiento del riesgo donde se modifica o implementan acciones frente al mismo para poder controlar la materialización de los riesgos o disminuir el impacto que podría provocar en caso de que se materializarse.

Palabras clave: impacto, oportunidad, residual, riesgo, sistema de gestión de riesgo.

\section{ABSTRACT}

The risk-based approach is one of the main changes of the new version of ISO 9001: 2015 standard, and in addition to the commitment of Senior Management, organizations are required to establish during the planning of the quality management system, actions that allow them to determine the risks and opportunities, in order to: ensure that the quality management system can achieve its expected results, increase desirable effects, prevent or reduce unwanted effects and achieve continuous improvement of their processes. In this article, a generic methodology is proposed to manage the strategic and operational risks of a company based on the understanding of the context and the identification of the needs and expectations of the interested parties. Through a sequence of stages it is proposed to address the risks and opportunities of an organization, based on the identification of the sources, causes and consequences that the completion of an inherent risk may bring, followed by the assessment through a qualitative, quantitative, semi-quantitative analysis or combination of these, according to the tool you want to use. Subsequently, having assessed the risk, it is evaluated using the defined strategies to identify the critical risks and their prioritization. Finally, the risk treatment process is continued where actions are modified or implemented in order to control the risks completion or reduce the impact that could be caused if it is completed.

Keywords: impact, opportunity, residual, risk, risk management system. 


\section{RESUMO}

A abordagem baseada em riscos é uma das principais mudanças da nova versão da norma ISO 9001:2015 e exige das organizações, além do compromisso da Alta Direção, estabelecer durante o planejamento do sistema de gestão da qualidade, as ações que viabilizem a determinação de riscos e oportunidades, visando: garantir que 0 sistema de gestão da qualidade conquiste seus resultados previstos, aumente os efeitos desejáveis, previna ou reduza os efeitos indesejáveis e alcance a melhoria contínua de seus processos. Neste artigo, propõe-se uma metodologia genérica para administrar os riscos estratégicos e operacionais de uma empresa partindo da compreensão do contexto e a identificação das necessidades e expectativas das partes em questão. Através de uma sequência de etapas pretende-se abordar os riscos e oportunidades de uma organização, partindo da identificação das fontes, causas e consequências que poderia trazer a concretização de um risco intrínseco, seguido da avaliação através de uma análise qualitativa, quantitativa, semiquantitativa ou a combinação de todas, conforme a ferramenta que quiser utilizar. A seguir, tendo analisado risco, avalia-se empregando as estratégias definidas para identificação e escolha dos riscos críticos. Por fim, segue o processo de tratamento do risco onde se alteram ou implementam as ações para evitar a concretização desses riscos ou diminuir o impacto que poderia ter se concretizados.

Palavras-chave: impacto, oportunidade, residual, risco, sistema de gestão de risco.

\section{INTRODUCCIÓN}

Con la evolución mundial del concepto de gestión de la calidad, los directivos de las empresas tomaron conciencia de que una forma de brindar confianza a sus clientes es mediante la implementación de un sistema de gestión que les permita asegurar que los productos o servicios ofrecidos cumplen con todas las necesidades y expectativas de los clientes. Por lo tanto, muchas compañías de distintos sectores de la economía a nivel mundial se han sumado a la implementación de un sistema de gestión de calidad, el cual está normalizado en su gran mayoría bajo el Organismo no gubernamental ISO, Organización Internacional para la Estandarización.

En la actualidad, el parámetro internacional más utilizado en esta temática es la norma ISO 9001, que a lo largo de su existencia ha pasado por diferentes versiones, correspondiente a los años 1994, 2000, 2008 y 2015. Cada vez que se lanza una nueva versión de la norma, esta trae consigo grandes cambios: en el año 2000 se introdujo el enfoque por procesos, en el año 2008 el centro es el cliente y se abre la puerta para la adaptación de requisitos con otras normas, para el año 2015 uno de los cambios más significativos es el nuevo enfoque basado en riesgos, el cual será objeto de estudio en la presente investigación.

El enfoque basado en riesgos sustituye a las acciones preventivas de la versión ISO 9001:2008, las cuales no tuvieron el efecto esperado, ya que en la mayoría de casos eran gestionadas por niveles inferiores en la organización y no abarcaban los factores que realmente afectaban a tales acciones a un nivel superior. Es decir que aun estando una empresa certificada en ISO 9001:2008 podría llegar a tener impactos negativos en su organización o en el sistema de gestión de la calidad, del tamaño de pérdida de la propiedad, iliquidez, pérdidas por demanda, malos negocios, corrupción, entre otros, ya 
que no se tenían identificados y controlados los riesgos asociados a la actividad de la organización.

Otro gran cambio que trae el enfoque basado en riesgos es que no solo considera los aspectos negativos, sino también los riesgos positivos, lo que definió como oportunidades, las cuales están orientadas a generar un gran impacto en la organización y en el sistema de gestión de la calidad, por ejemplo, apertura de mercados, nuevas prácticas, lanzamiento de productos, entre otros aspectos.

La Norma ISO 9000:2015 define el riesgo como una desviación de lo esperado, ya sea positivo o negativo. Esto nos da una idea de que la organización no solo se debe enfocar sus esfuerzos en los impactos negativos, sino también en las oportunidades potenciales. En el apartado A.4 de la norma ISO 9001:2015 se plantea que en el numeral 6.1 de esta misma norma la organización debe planificar acciones para abordar los riesgos, pero que sin embargo no hay ningún requisito en cuanto a métodos formales para la gestión del riesgo, las organizaciones pueden decidir si desarrollar o no una metodología de la gestión del riesgo más amplia de lo que requiere esta norma internacional.

La presente investigación tiene como objetivo definir una metodología idónea que permita identificar y controlar todos los riesgos y oportunidades de la organización de manera más práctica y de efectiva aplicación.

La literatura del enfoque basado en riesgos y las investigaciones realizadas en torno a esta temática son relativamente nuevas. Velásquez-Restrepo et al. (2017) planificaron y aplicaron estrategias orientadas a controlar posibles efectos adversos que surjan durante la atención a los usuarios, la calidad en el servicio y la seguridad del paciente. Dentro del trabajo efectuado se desarrolló un sistema de gestión del riesgo donde se identificaron cinco riesgos inherentes para el Laboratorio de Dermatopatología, seis para Otros Procesos Dermatológicos y ocho para la Unidad de Fotodermatología, los cuales fueron analizados y evaluados, luego de lo cual se implementaron los controles pertinentes.

Por otro lado, Cartin et al. (2014) realizaron un análisis de riesgo mediante el Análisis Modal de Efectos y Fallas (AMEF) incorporando en conjunto con el Análisis de Peligros y Puntos Críticos de Control (HACCP) para establecer la evaluación de riesgos en una maquiladora de pavo deshuesado. En este trabajo se calculó y se predijo, por medio de la significancia del riesgo, la importancia de establecer medidas preventivas en las etapas del proceso más vulnerables. El cálculo de la significancia se efectuó obteniendo el Índice de Criticidad (IC). Los puntos críticos de control se establecieron con la metodología del árbol de decisiones, que se enfocó en aquellas etapas que mostraron tener los valores más altos relativos al IC. Las principales causas posibles que generan una desviación de los puntos críticos de control fueron enlistadas y analizadas a través de un análisis causa-raíz, con el uso del diagrama de Ishikawa. La integración del análisis de riesgo dentro de un plan HACCP demostró proveer amplias ventajas que facilitan el control de los procesos de manufactura y el aseguramiento de la salud pública a los consumidores finales, al generar alimentos inocuos y de alta calidad, al tiempo que mejora el desempeño operacional del ciclo productivo.

Asímismo, Fernando F. Espinosa et al. (2012) describen el contexto de la organización e identifican las fuentes de riesgos en el desarrollo del proyecto de innovación, finalizando con la implementación de una matriz de valoración y jerarquización de las acciones que minimizan la posibilidad de que el riesgo sea realidad. 
De igual forma, Yudy Marcela y Christian Lochmüller proponen un esquema que describe la administración de riesgos basada en la gestión por procesos para las pymes comercializadoras en Medellín. Partieron de una encuesta donde identifican y analizan las estructuras y procesos que se aplican típicamente en estas comercializadoras para la gestión de los riesgos.

Por último, Muñoz y Cuadros, (2016) comparan modelos de gestión de riesgo como lógica difusa, FMEA, Octopus SME (método situacional de ingeniería), enfoque en gestión de riesgos (OSRIMA), PMI, MDD (desarrollo dirigido por modelos), RAM (Risk Analisis Method), ERP (Enterpreise Resources Planning) y método Schmidt de Brasil, como sugerencias para implementar en las pymes.

\section{METODOLOGÍA}

La elaboración de este artículo parte de la necesidad de definir herramientas para abordar los riesgos estratégicos y operativos de una organización, siendo estos un requisito de cumplimiento que exige la nueva actualización de la ISO 9001:2015. Haciendo uso de un enfoque descriptivo, la elaboración inicia con la búsqueda de diversas fuentes bibliográficas cuyo objetivo principal es la gestión de riesgo.

Figura 1. Pasos para desarrollar la investigación

\section{Selección de fuentes} bibliográficas

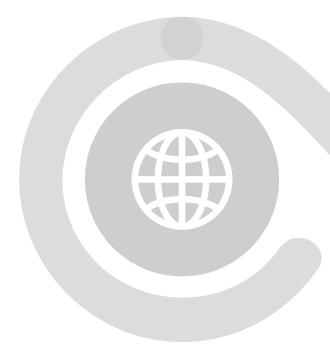

Planteamiento de las discusiones

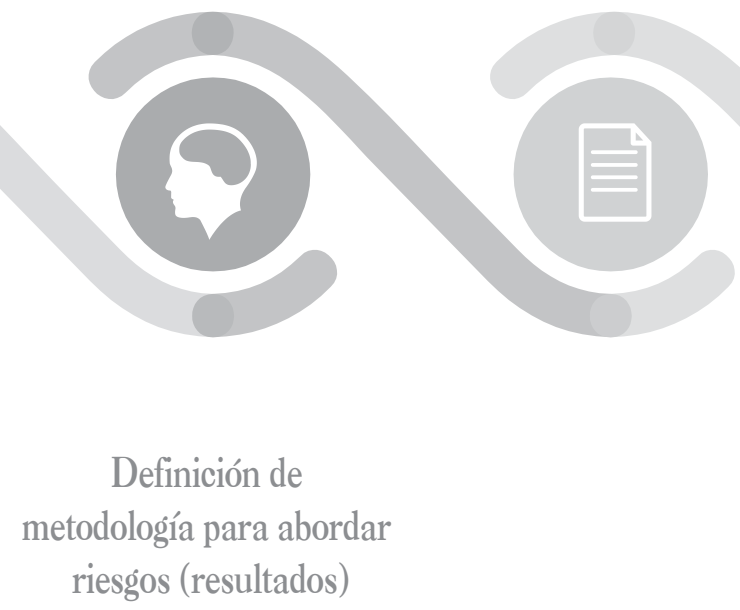

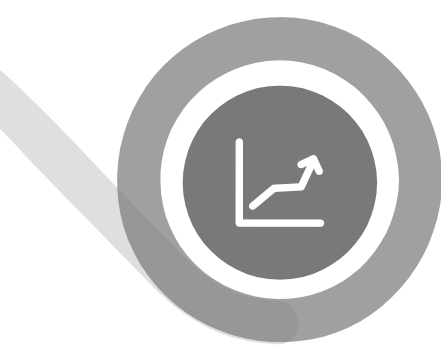

Conclusión y/o recomendación

Fuente: elaboración propia. 
En primer lugar (paso 1), se identificaron diversas fuentes bibliográficas por utilizar, las cuales tienen como factor principal la temática de riesgos. Posteriormente (paso 2), se realiza una compilación de las fuentes seleccionadas a través de la cual se extraen los aspectos más relevantes de los autores y que contribuyen a la definición de la metodología para gestionar riesgos, aquí se hace énfasis en la implementación de riesgos a través de la siguiente secuencia: identificación de riesgo, análisis de riesgo, valoración y tratamiento del riesgo. Resultado de lo anterior, se procede (paso 3) con el planteamiento de discusiones y, por último (paso 4), se definen las conclusiones entendiendo que abordar los riesgos pasa a ser uno de los elementos más importantes en la norma ISO 9001 versión 2015 y que aporta a la vez con la consecución de la mejora continua de los procesos de una compañía contribuyendo a la rentabilidad y sostenibilidad de esta.

\section{RESULTADOS Y DISCUSIÓN}

La norma ISO 9001 versión 2015 al incluir el enfoque basado en riesgo obliga a la organización a establecer acciones para identificar los riesgos que pueden afectar con sus objetivos estratégicos y operacionales. La gestión del riesgo no es un tema nuevo y tiene un posicionamiento propio en el desarrollo de la dirección debido a que sus fundamentos teóricos y metodológicos permiten dar un adecuado manejo a los riesgos.

Según Fernando et al. (2012), para aumentar las posibilidades de éxito de un sistema innovador es necesario en la organización tener una comprensión del riesgo potencial, evaluar sistemática y cuantitativamente estos riesgos, anticipando las causas y efectos posibles, y escoger entonces los métodos más apropiados para tratarlos. Para esta actividad existen diversas técnicas que se hablaran a lo largo de este capítulo.

Algunas de las características de la gestión de riesgos son las siguientes (Almuíñas, 2016):

- Es un método lógico y sistemático para identificar, evaluar y manejar los riesgos asociados a cualquier actividad, función o proceso, vinculado a la toma de decisiones.

- Se desarrolla como un proceso, con sus entradas (riesgos), transformación (análisis y tratamiento) y salidas (riesgos controlados).

- Los riesgos varían con el tiempo, aparecen unos y desaparecen otros, exigiendo de las organizaciones procesos constantes de planificación, seguimiento y evaluación.

- Los pasos básicos que sustentan la gestión de riesgos son la identificación, el análisis, la evaluación y el tratamiento de los riesgos (plan de riesgos), que se apoyan tanto en el análisis del contexto (externo e interno) y en la definición de parámetros para el manejo de los riesgos como en el desarrollo de acciones de comunicación, consulta, monitoreo y revisión.

- Una herramienta útil en la gestión de riesgos es el mapa de riesgos, ya que permite identificar, agrupar, ordenar, priorizar y evaluarlos para tomar decisiones sobre los que se van a asumir por la organización.

A continuación, se describen las etapas para la implementación de la gestión de riesgo en una organización. 


\section{Identificación del Riesgo}

La identificación del riesgo no es más que identificar las fuentes, causas y consecuencias que puede traer la materialización de un riesgo inherente, ya sea en el desarrollo de la producción de un producto, prestación de un servicio o en las actividades de la organización, que a su vez puedan causar el no cumplimiento de los objetivos estratégicos y operacionales establecidos.

El primer paso para esta etapa es establecer el contexto de la organización, puesto que genera un buen direccionamiento estratégico, aspecto que permite identificar las necesidades y expectativas de las partes interesadas, contribuir con la identificación y gestión del riesgo para cumplir con estas. Fontalvo \& De La Hoz (2018) y Velázquez et al. (2017) proponen una guía para identificar los riesgos, que consiste en responder a cuatro interrogantes que se muestran a continuación:

¿Qué puede suceder que afecte el logro de los objetivos del proceso u organización? Responder esta pregunta es identificar los riesgos o modos de falla reales o potenciales que pueden presentarse en la organización.

¿Por qué puede suceder? Responder esta pregunta implica identificar las causas que generan el riesgo o los modos de falla, los cuales pueden originarse por una 0 varias causas.

¿Agente generador del riesgo? Cuando se habla del agente generador, se identifica quién o qué está generando el riesgo. Este agente puede ser un software, una tecnología, la infraestructura de la clínica u hospital, talento humano, un proceso, etc.

¿Cuáles son los efectos más probables? Responder esta pregunta permite identificar los efectos o impactos que puede tener la materialización del riesgo. Es importante aclarar aquí que en esta etapa no se identifica el efecto más grave, sino el más probable, porque lo que busca la calificación de los riesgos y la ubicación en un mapa de calor es también tener una priorización de estos para su gestión, ya que los recursos de cualquier institución son limitados y se requiere identificar dónde es necesario enfocarlos para asegurar el logro de los objetivos organizacionales.

A partir de esta breve secuencia de preguntas es posible determinar los riesgos inherentes para luego clasificarlos y priorizarlos.

\section{Análisis de riesgo}

La finalidad de esta etapa es priorizar el riesgo mediante una valoración de este para luego poder establecer los tratamientos para mitigarlo. La ISO 31000 define esta etapa de la gestión del riesgo como el "análisis que brinda una entrada para la evaluación del riesgo y para las dimensiones sobre si es necesario o no tratar los riesgos y sobre las estrategias y métodos más adecuado[s] para su tratamiento". La entrada a la que hace referencia la norma es el nivel de riesgo o criticidad, que puede ser obtenido, ya sea por análisis cualitativo, cuantitativo, semicuantitativo o mediante la combinación de estos; donde el método para utilizar dependerá de la información, datos y recursos de los que se disponga y del propósito del análisis.

Existen diversas herramientas para la evaluación del riesgo donde relacionan la probabilidad o frecuencia de ocurrencia y el impacto. Velázquez et al. (2017) utilizaron la metodología de proyecto PMI que aplica un análisis semicuantitativo, donde el impacto del riesgo se evalúa cualitativamente como muy bajo, bajo, moderado, alto y muy alto, con una ponderación de 1 a 5 , respectivamente, y la frecuencia de ocurrencia como se muestra en la tabla 1. 
Tabla 1. Escala cualitativa de frecuencia de ocurrencia

\begin{tabular}{|c|c|c|}
\hline Escala cualitativa & Valor & Descripción \\
\hline Muy probable & 5 & $\begin{array}{l}\text { El evento ocurrirá con certeza o } \\
\text { cinco o más veces en el año }\end{array}$ \\
\hline Probable & 4 & $\begin{array}{l}\text { El evento probablemente } \\
\text { ocurre cuatro veces al año }\end{array}$ \\
\hline $\begin{array}{l}\text { Medianamente } \\
\text { probable }\end{array}$ & 3 & $\begin{array}{l}\text { El evento ocurre entre dos y } \\
\text { tres veces al año }\end{array}$ \\
\hline Poco probable & 2 & El evento ocurre una vez al año \\
\hline Improbable & 1 & $\begin{array}{l}\text { El evento ocurrirá solo en } \\
\text { circunstancias excepcionales }\end{array}$ \\
\hline
\end{tabular}

Fuente: Velásquez-Restrepo, Velásquez-Restrepo, Velásquez-Lopera \& Villa-Galeano (2017).
La calificación cualitativa del riesgo consta de un sistema de coordenadas de impacto y frecuencia como se muestra en la tabla 2.

Por otro lado, Consuegra (2015) en su investigación aplicaron la herramienta análisis modal de fallos y efectos (AMEF), que permite predecir desviaciones potenciales operativas causadas, que pueden poner en peligro el producto o proceso, de forma que lleve a la implementación de medidas correctivas o preventivas que minimicen sus efectos.

Tabla 2. Mapa de riesgos inherentes

\begin{tabular}{|l|l|l|l|l|l|}
\cline { 2 - 7 } \multicolumn{1}{|c|}{} & \multicolumn{5}{c|}{ Impacto } \\
\hline Frecuencia & Muy bajo & Bajo & Moderado & Alto & Muy alto \\
\hline Muy probable & Alto & Alto & Inaceptable & Inaceptable & Inaceptable \\
\hline Probable & Moderado & Alto & Alto & Inaceptable & Inaceptable \\
\hline $\begin{array}{l}\text { Medianamente } \\
\text { probable }\end{array}$ & Moderado & Moderado & Alto & Alto & Inaceptable \\
\hline Poco probable & Bajo & Bajo & Moderado & Alto & Alto \\
\hline Improbable & Bajo & Bajo & Moderado & Moderado & Alto \\
\hline
\end{tabular}

Fuente: Velásquez-Restrepo, Velásquez-Restrepo, Velásquez-Lopera \& Villa-Galeano (2017).

Esta herramienta utiliza tres parámetros de evaluación, los cuales son: la probabilidad de detección, el cual se define como la probabilidad de no detectar el error; la probabilidad de ocurrencia, que indica la frecuencia con la que puede ocurrir y, por último, el nivel de severidad al momento de materializarse el evento. Cada una se evalúa con valores enteros de 1 a 5 , siendo 1 la más baja calificación y 5 la más alta. El nivel de riesgo lo obtuvieron por medio del índice de criticidad, el cual es calculado mediante el uso de la ecuación 1 :

$$
I C=S \times 0 \times D \quad \text { Ec. }(1)
$$

Donde S, O y D son las variables severidad de riesgo, probabilidad de ocurrencia y probabilidad de detección. El valor del índice de criticidad del cual se toma la decisión de priorizar y clasificar el riesgo dependerá de lo establecido por la organización.

Otro método que cabe resaltar es la utilizada por Pérez \& Cruz (2014), quienes emplearon la metodología 
Enterprise Risk Management (ERM), definida como "la gestión de riesgos empresariales que consiste en un proceso de identificación y análisis de riesgo desde una perspectiva amplia e integral de la compañía”. Esta herramienta usa como parámetros la viabilidad (hace referencia a la frecuencia) y el impacto, ambas con valoración de 1 a 5. Mediante esta herramienta se pueden hacer dos análisis, el primero cualitativo en el que se ubicaban en el cuadro de evaluación de riesgo que se muestra en la tabla 3, donde se consideran cuatro cuadrantes, considerando el eje $\mathrm{Y}$ como viabilidad y eje $\mathrm{X}$ como impacto.

El segundo análisis es cuantitativo, donde la valoración del riesgo se obtiene por medio de la ecuación 2, y nuevamente el criterio para priorizar lo establece la organización:

Tabla 3. Cuadro de evaluación de riesgo

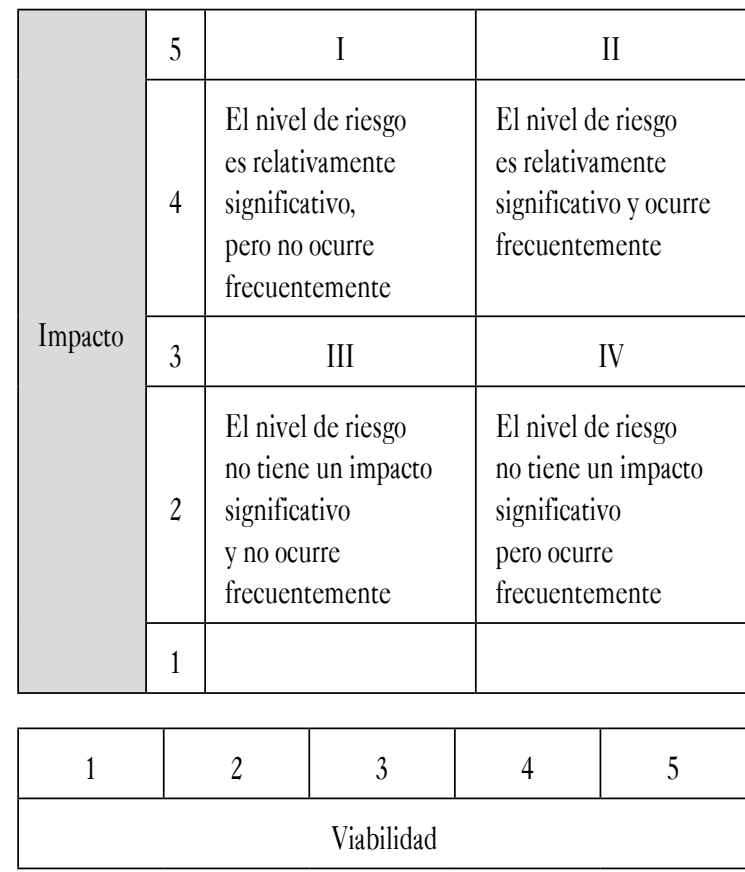

Fuente: Pérez-Castaneda \& Cruz-Ramírez (2014).

$$
. V R=V x I \quad \text { Ec. }(2)
$$

Como se puede evidenciar, existen numerosas herramientas que van a depender, como se dijo anteriormente, de la información que se tenga. Para el análisis de riesgos estratégicos es aconsejable utilizar la técnica DAFO/FODA, que permite analizar las debilidades, fortalezas, amenazas y oportunidades. Esta es una de las técnicas más empleadas en la planeación estratégica, en especial para la determinación de la posición estratégica de la empresa. Por lo demás, es una importante herramienta de apoyo para la toma de decisiones generalmente usadas para analizar de manera sistemática los ambientes interno y externo de una organización (Nikulin \& Becker, 2015).

\section{Evaluación del riesgo}

En esta etapa es importante establecer el efecto que determinadas situaciones y eventos pueden tener en consecución de los objetivos empresariales, por lo que resulta necesario evaluarlos teniendo en cuenta su impacto económico, así como su probabilidad de ocurrencia. Por lo anterior, es necesario emplear técnicas cuantitativas y cualitativas, centrándose en primera medida en el riesgo inherente 0 asociado a la inmediatez de las acciones ejecutadas sin el establecimiento de mecanismos de control, y luego, el riesgo residual que resulta posterior de la implementación de las medidas de control (Rincón, 2018).

Con base en lo anterior, podemos afirmar que la entrada para la evaluación es el nivel o valoración de riesgo, dependiendo de la herramienta utilizada, puesto que a partir de este se puede determinar cuál de los riesgos identificados son críticos, cuáles se deben priorizar y qué acciones y tratamiento se deben tomar al respecto.

Existen cuatro estrategias que son muy útiles al momento de evaluar el riesgo, las cuales son (Jhuéz, 2018). 
- Supresión del riesgo: no es lo más habitual, pero a veces las organizaciones logran que desaparezcan los riesgos asociados a sus procesos. Esto se consigue cuando la labor de previsión se ha implementado de forma exitosa, obteniendo información adicional, adquiriendo apoyo de expertos, añadiendo recursos adicionales o modificando los elementos de la planificación, entre otros elementos.

- Mitigación del riesgo: es una estrategia de gestión de riesgos que consiste en reducir la probabilidad o el impacto de un riesgo sobre la organización. Es decir, que si llega a producirse sus efectos serán mucho menores que si no se hubiesen adoptado medidas al respecto. Esta opción se usa sobre todo en aquellos casos en que los riesgos son inevitables o no dependen de la empresa en sí misma. La clave para una acertada mitigación del riesgo está en las acciones.

- Explotación del riesgo: no todos los riesgos son negativos. Algunas veces, su irrupción es una oportunidad para las organizaciones. Cuando eso ocurre, en vez de mitigarla o eliminarla, la estrategia de la empresa debe centrarse en sacar el máximo provecho de la circunstancia. Un riesgo con efectos positivos se puede potenciar gracias a la designación de más personal cualificado, mayor apoyo económico o una adaptación a la planificación realizada al inicio.

- Aceptación del riesgo: en estos casos, se trata de riesgos que no suponen mayores impedimentos para la consecución de los objetivos y que, por tanto, pueden convivir con la empresa. Pero no se trata de una actitud resignada. Por el contrario, implica la elaboración de un plan de contingencia para, de este modo, adaptar el riesgo a las actividades de las empresas. Por ejemplo, las compañías que operan en zonas montañosas y con una alta probabilidad de sismos desarrollan toda una política de emergencia en torno a la evacuación y la asistencia en casos de emergencia.

\section{Tratamiento de riesgo}

El objetivo de esta etapa, de acuerdo con la norma ISO 31000 es el proceso de modificar el riesgo e implementar acciones frente al mismo. Los tratamientos pueden modificar los riesgos existentes o crear nuevos riesgos, así como suministrar controles o modificar los existentes. Por lo tanto, las acciones de tratamiento pueden implicar:

- Evitar el riesgo decidiendo no iniciar o continuar la actividad que lo originó

- Tomar o incrementar el riesgo, con el fin de perseguir la oportunidad

- Retirar la fuente de riesgo

- Cambiar la probabilidad

- Cambiar las consecuencias

- Compartir el riesgo con una o varias de las partes (incluyendo los contratos y la financiación de riesgo)

- Retener el riesgo a través de la decisión informada

El tratamiento del riesgo, de acuerdo con la ISO 31000, debe ser un proceso cíclico, en donde se debe valorar el tratamiento del riesgo, la decisión de si los niveles de riesgos son tolerables, la generación de nuevos tratamientos y la valoración de la eficacia de estos.

Partiendo de la estrategia de supresión del inciso anterior, nos damos cuenta de que no es común ver que un riesgo se elimina puesto que siempre se tendrá un 
residual. Este puede definirse como la línea que separa a la organización de la seguridad total de sus procesos, es decir, que siempre se va a mitigar mediante la búsqueda de estrategias que permita establecer acciones para poder contralar su materialización o disminuir el impacto que podría provocar en caso de materializarse. Este es el objetivo principal de esta etapa, mirar si el riesgo residual es permisible para el alcance de los objetivos, y de no ser así entraríamos en el ciclo propuesto anteriormente.

Una vez cumplidas todas estas etapas y siendo no menos importante en la gestión de riesgo, se debe realizar el proceso de seguimiento y monitoreo de los riesgos; de nada sirve identificar todas las fuentes que afectan mis actividades y objetivos, establecer acciones de control y tomar tratamientos si no se va a monitorear y evaluar la eficacia de las acciones planteadas.

El propósito de esta investigación fue proponer y describir una metodología que permita a las organizaciones tomar acciones para abordar los riesgos y oportunidades a partir de la comprensión del contexto de la compañía y necesidades y expectativas de sus partes interesadas, en busca de dar cumplimiento al requisito 6.1 de la norma ISO 9001:2015.

A través del estudio y análisis de los artículos citados se comprobó que cada una de estas metodologías utilizadas son efectivas de acuerdo con el contexto de la organización, en todos los casos de aplicación se logró identificar, analizar y controlar los riesgos, tomando acciones que permitan, por un lado, minimizar la probabilidad de que los impactos negativos se materialicen y, por otro, materializar las oportunidades o riesgos positivos.

La presente metodología propuesta es genérica para todo tipo de organización y contexto, y de fácil interrelación con otros métodos y herramientas más robustas, teniendo en cuenta que no todas las organizaciones tienen el mismo nivel de riesgo e incertidumbres. Se considera que al ser aplicada la metodología planteada podría alcanzar resultados satisfactorios para que una organización administre eficientemente sus riesgos y realice un seguimiento y monitoreo de los controles establecidos, ya que reúne y se sustenta en diferentes investigaciones y casos de éxito relacionados con esta temática.

\section{CONCLUSIONES}

El artículo aporta una metodología y un conjunto de soluciones que permitirán a las diferentes organizaciones desarrollar adecuadamente la gestión del riesgo, así mismo se contribuye a la literatura en torno a esta temática, generando material de estudio que reúne información de diversas fuentes bibliográficas confiables. A partir de los resultados podemos concluir los aspectos que se mencionan a continuación.

Abordar la gestión del riesgo permite identificar y tratar aquellas actividades que pueden tener impacto negativo en el alcance de los objetivos planteados por la organización, que pueden estar relacionados con el cumplimiento de las necesidades y expectativas de todas las partes interesadas; objetivos que pueden ser tanto estratégicos como operacionales establecidos en el sistema de gestión de calidad.

Para una correcta identificación de los riesgos primero se debe determinar o conocer el contexto de la organización, identificar las necesidad y expectativas de las partes interesadas. Una vez hecho esto, se identifican estas acciones teniendo en cuenta los factores que puedan afectar el logro de los objetivos del proceso u organización, por qué puede suceder, qué puede provocarlo y qué impacto traerá esta acción si se materializa. 
En el análisis de riesgo existen diversas herramientas que ayudan a obtener el nivel o valoración del riesgo, como es la metodología de proyecto PMI, análisis modal de fallas AMEF, Enterprise Risk Management (ERM), entre otras, las cuales se basan en la probabilidad o frecuencia de ocurrencia, y el impacto que causaría.

En la etapa de evaluación se priorizan los riesgos a partir del nivel o valoración del riesgo, y se toman las acciones pertinentes acorde a cuatro estrategias planteadas: supresión, mitigación, explotación y aceptación del riesgo.

En la etapa de tratamiento se evalúan las acciones implementadas y se escoge como objeto de estudio los riesgos residuales para cada actividad de peligro identificada. Lo ideal es que el riesgo residual sea el mínimo o tolerable.

Para la aplicación de esta metodología se recomienda seguir cada una de las etapas propuestas, identificando en cada una de las mismas la o las herramientas que más se ajusten al contexto y nivel de riesgo de la organización.

\section{REFERENCIAS}

Instituto Colombiano de Normas Técnicas y Certificación (2015). NTC-ISO 9000:2015. Sistemas de gestión de la calidad. Fundamentos y vocabulario. Colombia: Icontec.

Instituto Colombiano de Normas Técnicas y Certificación (2015). NTC-ISO 9001:2015. Sistemas de gestión de la calidad. Requisitos. Colombia: Icontec.

Velásquez-Restrepo, P. A., Velásquez-Restrepo, S. M., Velásquez-Lopera, M. \& Villa-Galeano, J. (2017). Implementación de la gestión de riesgo en los procesos misionales de la Sección de Dermatología de la Universidad de Antioquia (Medellín, Colombia) siguiendo las directrices de la norma ISO 9001:2015. Revista gerencia y politicas de salud, 16(33), 78-101. DOI https://doi.org/10.11144/Javeriana.rgps16-33. igrp

Cartin, A., Villarreal-Tello, A. \& Morera, A. (2014). Implementación del análisis de riesgo en la industria alimentaria mediante la metodología AMEF: enfoque práctico y conceptual. Revista de medicina veterinaria, 27, 133-148. DOI https://doi.org/10.19052/ mv.3030

Espinosa, F., Días, A. \& Salinas, E. (2012). Un procedimiento para evaluar el riesgo de la innovación en la gestión del mantenimiento industrial. Revista Chilena de ingeniería, 20(2), 242-254. DOI http://dx.doi. org/10.4067/S0718-33052012000200011

Hernández-García, Y. \& Lochmüller, C. (2012). Aplicación de la gestión de riesgos en los principales procesos de una pyme comercializadora. Revista Soluciones de Postgrado EIA, 9, 143-165. Recuperado de http://hdl.handle.net/11190/708

Muñoz-Holguín D. \& Cuadros-Mejía, A. (2016). Comparación de metodologías para la gestión de riesgos en los proyectos de las pymes. Revista ciencias estratégica, 25(38), 319-338. DOI http://dx.doi. org/10.18566/rces.v25n38.a4

Almuíñas, J. L. (2016). La gestión de riesgos: una alternativa para apoyar la gestión universitaria con enfoque estratégico. Universidad de La Habana, Revista Cubana de Educación Superior, 35, 2.

Fontalvo, T. \& De La Hoz, E. (2018). Diseño e Implementación de un sistema de gestión de la calidad ISO 9001:2015 en una universidad colombiana. Revista 
formación universitaria, 11(1), 35-44. DOI http:// dx.doi.org/10.4067/S0718-50062018000100035

Instituto Colombiano de Normas Técnicas y Certificación (Icontec). NTC-ISO 31000-2011: gestión del riesgo, principios y directrices. Colombia: Icontec.

Consuegra-Mateus, O. (2015). Metodología AMFE como herramienta de gestión de riesgo en un hospital universitario. Revista cuadernos latinoamericanos de administración, 11(20), 37-50. DOI: https://doi. org/10.18270/cuaderlam.v11i20.627

Pérez-Castañeda, S. \& Cruz-Ramírez, D. (2014). Propuesta de evaluación de riesgos empresariales en microempresas manufactureras. Revista de la facultad de ciencias contables, 22(41), 165-174. DOI https:// doi.org/10.15381/quipu.v22i41.10082

Nikulin, C. \& Becker, G. (2015). Una metodología sistémica y creativa para la gestión estratégica: Caso de Estudio Región de Atacama-Chile. Journal of technology management \& innovation, 10(1). 127-144. Doi http://dx.doi.org/10.4067/S071827242015000200009

Rincón-Ramón, A. (2006). COSO II y la gestión integral de riesgo del negocio. Recuperado de http://pdfs.wke. es/6/6/7/3/pd0000016673.pdf.

Jhuéz, J. (2018). Metodologías para gestión del riesgo. España: Universidad Politécnica de Catalunya. Recuperado el 26 de septiembre de 2018, de https://capacitacioncgr.jovenclub.cu/wp-content/ uploads/2018/05/Metodologia-para-la-Gestiondel-Riesgo.pdf 